\title{
Vection in depth during treadmill walking
}

\author{
April Ash ${ }^{1}$, Stephen Palmisano ${ }^{1}$, Deborah Apthorp², Robert S Allison ${ }^{3}$ \\ ${ }^{1}$ School of Psychology, University of Wollongong, Wollongong, Northfields Avenue, NSW 2522, \\ Australia; e-mail: aea404@uowmail.edu.au; ${ }^{2}$ Research School of Psychology, College of \\ Medicine, Biology and Environment, Australian National University, ACT 0200, Australia; \\ ${ }^{3}$ Department of Computer Science and Engineering, York University, 4700 Keele Street, \\ Toronto, ON M3J 1P3, Canada \\ Received 14 January 2013, in revised form 2 May 2013
}

\begin{abstract}
Vection has typically been induced in stationary observers (ie conditions providing visualonly information about self-motion). Two recent studies have examined vection during active treadmill walking - one reported that treadmill walking in the same direction as the visually simulated selfmotion impaired vection (Onimaru et al, 2010 Journal of Vision 10(7):860), the other reported that it enhanced vection (Seno et al, 2011 Perception 40 747-750; Seno et al, 2011 Attention, Perception, \& Psychophysics 73 1467-1476). Our study expands on these earlier investigations of vection during observer active movement. In experiment 1 we presented radially expanding optic flow and compared the vection produced in stationary observers with that produced during walking forward on a treadmill at a 'matched' speed. Experiment 2 compared the vection induced by forward treadmill walking while viewing expanding or contracting optic flow with that induced by viewing playbacks of these same displays while stationary. In both experiments subjects' tracked head movements were either incorporated into the self-motion displays (as simulated viewpoint jitter) or simply ignored. We found that treadmill walking always reduced vection (compared with stationary viewing conditions) and that simulated viewpoint jitter always increased vection (compared with constant velocity displays). These findings suggest that while consistent visual-vestibular information about self-acceleration increases vection, biomechanical self-motion information reduces this experience (irrespective of whether it is consistent or not with the visual input).
\end{abstract}

Keywords: vection, locomotion, treadmill, optic flow, multisensory

\section{Introduction}

A compelling visual illusion of self-motion, known as vection, can be induced when a large optic flow pattern is presented to a physically stationary observer. Despite the apparent dominance of vision in this particular situation (Johansson 1977; Lee and Lishman 1975; Lishman and Lee 1973), a number of nonvisual senses also provide useful information, particularly about active self-acceleration (Benson 1990; Howard 1982; Siegler et al 2000). The vestibular system is more sensitive to high temporal frequency self-motion (ie greater than $1 \mathrm{~Hz}$ ) than vision and is often thought to govern the perception of both linear and angular self-accelerations (Berthoz et al 1975, 1979; Diener et al 1982; Howard 1986; Melvill-Jones and Young 1978). However, while the threshold for detecting whole-body oscillation is almost ten times greater for those without functional labyrinths (Walsh 1961), the vestibular system cannot distinguish between moving at a constant velocity and remaining stationary (Benson 1990; Lishman and Lee 1973). In addition to the vestibular system, the somatosensory system and proprioception also provide useful biomechanical information about active self-motion based on the pressure and shear forces acting on the skin and the inertia of the limbs, respectively (Lee and Lishman 1975; Lishman and Lee 1973; Mergner and Rosemeier 1998). Despite this, most previous studies have examined vection in physically stationary observers (Palmisano et al 2000, 2003, 2008). Only a few studies have examined vection in actively moving observers in which the nonvisual senses to self-motion have been systematically stimulated (eg Kim and Palmisano, 2008, 2010). The current study examines the effect of treadmill walking on vection induction. 


\subsection{Effect of simulated viewpoint jitter on vection}

Traditionally, it was thought that conflicts between the above-mentioned sensory systems (particularly visual-vestibular conflicts) would impair vection (see Zacharias and Young's 1981 sensory conflict theory). For example, when a stationary observer views an optic flow display simulating constant velocity forwards self-motion, the characteristic delay in vection onset (typically a few seconds) was thought to be due to the transient conflict between the visual and vestibular systems - with the visual system indicating that the observer is moving, while the vestibular system would register that he or she is physically stationary. However, more recent research (Palmisano et al 2000, 2003, 2008, 2011) has consistently shown that adding visually simulated viewpoint jitter/oscillation to a display increases vection in stationary observers, even though this situation would produce sustained visual-vestibular conflict - the visual system would indicate horizontal and vertical acceleration throughout the trial, while the vestibular system would indicate that the observer is physically stationary (ie no self-acceleration).

More recently, vection studies have generated simulated viewpoint jitter/oscillation by tracking seated subjects' physical head motions while they viewed a self-motion display (these fore-aft or left-to-right head motions were typically in time with a computer-controlled metronome). In such situations the motion of the display concomitant with the head can be ecological or not. Consider an observer moving their head in a plane parallel to a nearby stationary display simulating a window. Ecologically, the world is stable and 'out there'. Therefore, owing to parallax, images of the world beyond the window should move with respect to the window (display) frame in the same direction as the head motion. Motion in the opposite direction of the head indicates an unstable world or that the environment is in front of the window, both generally nonecological self-motion scenarios. Moving fore-aft should cause expansion and contraction of the environment including the display. Contraction and expansion of the image within the display while moving forward and backward, respectively, is similarly nonecological.

Previous active seated vection studies (eg Ash and Palmisano 2012; Kim and Palmisano 2008, 2010; Ash et al 2011a, 2011b) had subjects move their heads from side to side or fore-aft, and used these tracked head position changes to move the virtual camera for the self-motion display to generate ecological or nonecological jittering patterns of multisensory stimulation. These studies have tended to find no difference between 'ecological' and 'nonecological' jittering patterns of multisensory stimulation during active head movements. A recent study by Ash et al (2011a), however, showed an advantage for ecological (compared with nonecological) simulated viewpoint jitter in some self-motion situations.

\subsection{Vection during treadmill walking}

To date, only two studies have examined the effect of active treadmill walking on vection. In the first of these studies Onimaru et al (2010) presented expanding or contracting optic flow patterns (simulating forwards or backwards self-motion at $2 \mathrm{~km} \mathrm{~h}^{-1}$ ) to their subjects as they walked either forward or backward on an omnidirectional treadmill at $2 \mathrm{~km} \mathrm{~h}^{-1}$. They found that vection latencies were longer when subjects physically walked in the same direction as the simulated self-motion compared with when they walked in the opposite direction. In the second study, by Seno et al (2011a), subjects viewed upward, downward, leftward, rightward, forward (expanding optic flow), or backward (contracting optic flow) self-motion displays while they were either stationary (no locomotion conditions) or walking forwards on a unidirectional treadmill at $2 \mathrm{~km} \mathrm{~h}^{-1}$ (with locomotion conditions). Unlike for Onimaru et al (2010), the display was simulated at a much faster speed $\left(16 \mathrm{~m} \mathrm{~s}^{-1}\right.$ or $\left.57.6 \mathrm{~km} \mathrm{~h}^{-1}\right)$ than the treadmill belt speed. Vection induced while viewing upward, downward, rightward, and leftward self-motion displays resulted in longer latencies, shorter durations, and smaller 
magnitude ratings during treadmill walking than stationary viewing conditions. However, of particular relevance to the current study was: (i) backward self-motion displays (contracting optic flow) produced better vection than forward self-motion displays (expanding optic flow) when the observer was stationary; but, (ii) contrary to Onimaru et al (2010), forward self-motion displays (which provided consistent multisensory information about the direction of self-motion) produced better vection than backward self-motion displays (where the multisensory information about self-motion direction was inconsistent) during treadmill walking. Furthermore, viewing forward self-motion displays during forward treadmill walking also resulted in better vection than viewing these same displays while stationary.

\subsection{Redundant multisensory information during treadmill walking}

A number of seated (Li et al 2009; Yeung and Li 2013) and treadmill walking studies (Durgin and Gigone 2007; Durgin et al 2005) have found reductions in visually perceived object motion during redundant multisensory situations. These previous findings appear to be generally consistent with Barlow's inhibition theory, which asserts that: (i) there is a reduction in the salience of retinal motion during positively correlated (as opposed to uncorrelated or negatively correlated) multisensory events because these situations provide redundant information about self-motion; and (ii) redundant retinal motion signals are suppressed by nonvisual signals to minimise their salience. More recently, Durgin (2009) extended on Barlow's inhibition theory, proposing that reductions in visually perceived display speed play a functional role in preserving the ability to discriminate changes in speed while an observer is walking forward and viewing an expanding optic flow display (this finding has since been replicated by Souman et al 2010). Previous vection studies have shown that simulated display speed (Brandt et al 1973; Dichgans and Brandt 1978) and the perceived speed of self-motion (Apthorp and Palmisano 2012; Palmisano 2002) are positively related to vection. Thus, it is possible that reported reductions in visually perceived display speed during forward treadmill walking while viewing an expanding optic flow display could also affect perceptions of self-motion.

Importantly, Durgin et al (2005) showed that the visual consequences of head movements during forward treadmill walking were not responsible for these reductions in visually perceived display speed while viewing expanding optic flow. Durgin et al (experiment 2) recorded physical head movements while subjects walked forward on a treadmill and later on played the resulting 'jittering optic flow' displays back to their now stationary subjects (these displays simulated both forwards self-motion and the bob, sway, and lunge head motions). Their subjects were also shown purely radial (ie 'nonjittering') self-motion displays on half of their stationary viewing trials. The reductions in perceived display speed produced by treadmill walking compared with stationary viewing were the same regardless of whether the stationary subjects viewed smooth or jittering optic flow. Furthermore, Durgin and colleagues (Durgin and Gigone 2007; Durgin et al 2005) also showed that reductions in the visually perceived display speed during treadmill walking (compared with stationary viewing) are proportional to physical walking speed, with the greatest reductions occurring for faster treadmill belt speeds and speeds closest to normal walking speeds.

\subsection{Object-motion versus self-motion perception}

Barlow's (1990) theory does not explicitly state whether redundant sensory information generates reductions in visually perceived object motion, visually perceived self-motion, or both. Optic flow can arise either from self-motion or from object or scene motion and/or a combination thereof. If the observer perceives less object or scene motion induced by optic flow (despite there being no actual change in the optic flow itself), then this might be due to the optic flow being perceived as resulting more from self-motion than object or scene motion. 
A more compelling experience of vection occurs when subjects perceive an optic flow display as resulting more from self-motion than object or scene motion. If reductions in visual motion affect only visually perceived object motion, then Barlow's (1990) theory might also predict an increase (rather than a decrease) in vection during consistent multisensory self-motion situations (compared with inconsistent multisensory situations), as was found by Seno et al (2011a). On the other hand, if reductions in visual motion also affect visually perceived self-motion, then a modified version of Barlow's theory might predict a decrease in vection during consistent multisensory self-motion situations (compared with inconsistent multisensory situations), as was found by Onimaru et al (2010).

\subsection{The current study}

Our experiments will further examine the effect of treadmill walking on vection in depth. In experiment 1 we compared the vection induced when subjects either walked forward on a treadmill or viewed displays while stationary. During treadmill walking conditions tracked linear and rotary physical head movements were either updated into the self-motion display (as ecological simulated viewpoint jitter) or simply ignored (the display simulating smooth forwards self-motion). Passive conditions were playbacks of the optic flow display shown or generated in the previous active treadmill walking trials - in this case now viewed while standing still. Previous treadmill experiments by Seno et al (2011a) and Onimaru et al (2010) examined only nonjittering displays (although since their subjects' heads physically moved during treadmill walking, this would have generated some additional nonecological 2 -D visual jitter). On the basis of previous seated vection studies (Ash and Palmisano 2012; Ash et al 2011a, 2011b; Kim and Palmisano 2008, 2010; Palmisano et al 2003, 2008), we expected jittering displays to increase vection compared with nonjittering displays when subjects were physically stationary. However, the effect of simulated viewpoint jitter on vection during treadmill walking is currently unknown. This really depends on whether visually perceived object motion, visually perceived self-motion, or both are reduced during treadmill walking. It is also possible that, on the basis of a modified version of Barlow's (1990) theory, displays with simulated viewpoint jitter might show greater reductions in vection during forward treadmill walking than nonjittering displays (compared with viewing the same displays while stationary) because the former condition would provide additional consistent or redundant visual information about self-motion. Alternatively, as simulated viewpoint jitter has been shown to provide a robust advantage for vection, it is possible that this 3-D perspective display jitter might continue to increase vection compared with constant velocity optic flow displays, even if there are significant reductions in vection during treadmill walking.

For the first time in a treadmill study we also investigated the effect of treadmill and/or display forward speed using two treadmill/display forward speeds $\left(4 \mathrm{~km} \mathrm{~h}^{-1}\right.$ and $\left.5 \mathrm{~km} \mathrm{~h}^{-1}\right)$ typical of human walking. Previous vection studies have examined only very slow treadmill belt speeds $\left(2 \mathrm{~km} \mathrm{~h}^{-1}\right)$ that were outside the range of normal walking speeds [the range of speeds for which Durgin (2009) proposes reductions in visually perceived speed are greatest]. Furthermore, Seno et al (2011a) used a physical display speed that was much faster than the physical treadmill belt speed. Thus, in the current study we were interested in: (i) examining walking speeds that were within the range of normal human walking speeds; and (ii) matching the simulated display (simulated translation) and treadmill belt speed to provide consistent visual and nonvisual information about self-motion. As increases in both simulated display speed (Brandt et al 1973; Dichgans and Brandt 1978) and the perceived speed of self-motion (Apthorp and Palmisano 2012; Palmisano 2002) are suggested to increase vection (at least up to a certain point), we might expect faster treadmill/display speeds to result in stronger, more compelling vection than slower treadmill/display speeds. 
Experiment 2 was specifically designed to study (and ideally resolve) the apparent contradiction between Ominaru et al's (2010) and Seno et al's (2011a) treadmill findings for vection. Observers viewed either expanding or contracting optic flow displays while walking forward on a treadmill to determine whether the visually simulated direction of self-motion (which was either consistent or inconsistent with the direction of the treadmill belt) affected vection. If redundant multisensory information about self-motion reduces vection (similar to Onimaru et al 2010), then contracting optic flow should induce stronger vection than expanding optic flow during forward treadmill walking. Alternatively, on the basis of Seno et al (2011a), if redundant multisensory information about self-motion enhances vection, then we might find that expanding optic flow produces stronger vection than contracting optic flow during forward treadmill walking.

\section{Experiment 1: vection in depth during active and simulated forward treadmill walking at two different speeds}

Here we compared vection in depth during treadmill walking (consistent or inconsistent multisensory information about self-motion) with viewing a playback of these displays while stationary (vision-only information about self-motion). For the first time in a treadmill walking study we: (i) compared the vection induced by jittering (generated by the subject's own head movements while walking) and nonjittering optic flow displays; and (ii) used two different display speeds simulating forward self-motion at either $4 \mathrm{~km} \mathrm{~h}^{-1}$ or $5 \mathrm{~km} \mathrm{~h}^{-1}$, which are both within the range of average human walking speeds. Unlike the current experiment, previous vection studies: (i) did not update subjects' physical head movements generated during treadmill walking into the self-motion display as consistent simulated head movements; and (ii) used only a single treadmill speed $\left(2 \mathrm{~km} \mathrm{~h}^{-1}\right)$ that was not very representative of normal human walking speeds. During treadmill walking conditions in the current experiment the treadmill belt speed was adjusted to match the simulated speed of self-motion represented by the expanding radial flow display.

\subsection{Method}

2.1.1 Subjects. Twenty undergraduate psychology students (sixteen females and four males; mean age $=23.05$ years, $\mathrm{SD}=2$ years) at the University of Wollongong received course credit for their participation in this experiment. All subjects had normal or corrected-to-normal visual acuity and no self-reported vestibular or neurological impairments. The Wollongong University Ethics Committee approved the study in advance and each subject provided written informed consent before participating in the study.

2.1.2 Displays and apparatus. A Mitsubishi Electric (Model XD400U) colour DLP data projector $(1024 \times 768$ pixel resolution; refresh rate $=60 \mathrm{~Hz})$ was used to rear project computer-generated displays (Dell Optiplex GX620 PC) onto a flat projection screen (1.48 m wide $\times 1.20 \mathrm{~m}$ high). Displays were programmed in Python using the Visual Python graphics library (Version 2.6) and consisted of 600 randomly positioned blue spheres/dots $\left(3.2 \mathrm{~cd} \mathrm{~m}^{-2}\right)$ on a black background $\left(0.1 \mathrm{~cd} \mathrm{~m}^{-2}\right)$. These displays simulated constant velocity forward selfmotion through a 3-D environment that was 173 units wide by 130 units high and 300 units $(\sim 3 \mathrm{~m})$ deep (object density was one dot per cube unit). As objects in this 3-D environment disappeared off the front edge of the screen, they were replaced at the same horizontal and vertical coordinates at the opposite end of space. We also used a motorised treadmill (ProForm PF 4.0) and Logitech head-tracking system during active treadmill walking conditions (see figure 1), both of which were modified to receive input from the same computer used to generate optic flow displays - this input was used to start, stop, and control the treadmill's belt speed. The treadmill belt and the head tracker were also programmed using Python-based software. 
Displays were viewed by subjects while either walking on a moving treadmill or standing on a motionless treadmill. The treadmill placed subjects at a distance of approximately $0.9 \mathrm{~m}$ from the display screen, so that the display subtended an area approximately $79 \mathrm{deg}$ wide by $67 \mathrm{deg}$ high of visual angle. All visual displays simulated forward motion (an expanding optic flow pattern) at either $4 \mathrm{~km} \mathrm{~h}^{-1}$ or $5 \mathrm{~km} \mathrm{~h}^{-1}$. During active treadmill walking conditions the treadmill was programmed to match the simulated forward speed of the display for each trial (ie the display speed increased proportionally with the treadmill belt speed).

Subjects wore a worker's helmet that was fitted with the 3-D Logitech head-tracking system receiver ( 6 degrees of freedom sensing for $x, y, z$, pitch, roll, and yaw). The transmitter of this ultrasonic head-tracking system was mounted on the ceiling of the laboratory directly above the treadmill, and subjects were positioned under this transmitter at the beginning of each trial. Importantly, the head-tracking data were recorded in real time by the same computer used to generate the optic flow displays, and the software used to generate these displays had real-time access to the head-tracking data ( $\sim 60 \mathrm{~ms}$ end-to-end system lag). This system was used to track subjects' active head movements during treadmill walking, and then these head position and orientation changes were: (i) directly incorporated into the computer-generated self-motion displays during active conditions (as ecological simulated viewpoint jitter); (ii) played back as simulated viewpoint jitter to stationary subjects during passive viewing conditions; or (iii, iv) ignored by the computer generated self-motion display during other ('nonjittering') active or passive viewing conditions. In addition to their constant velocity forward self-motion component, active or passive 'jittering' displays also simulated head translations along the horizontal, vertical, and depth axes, and simulated roll, pitch, and yaw head rotations.

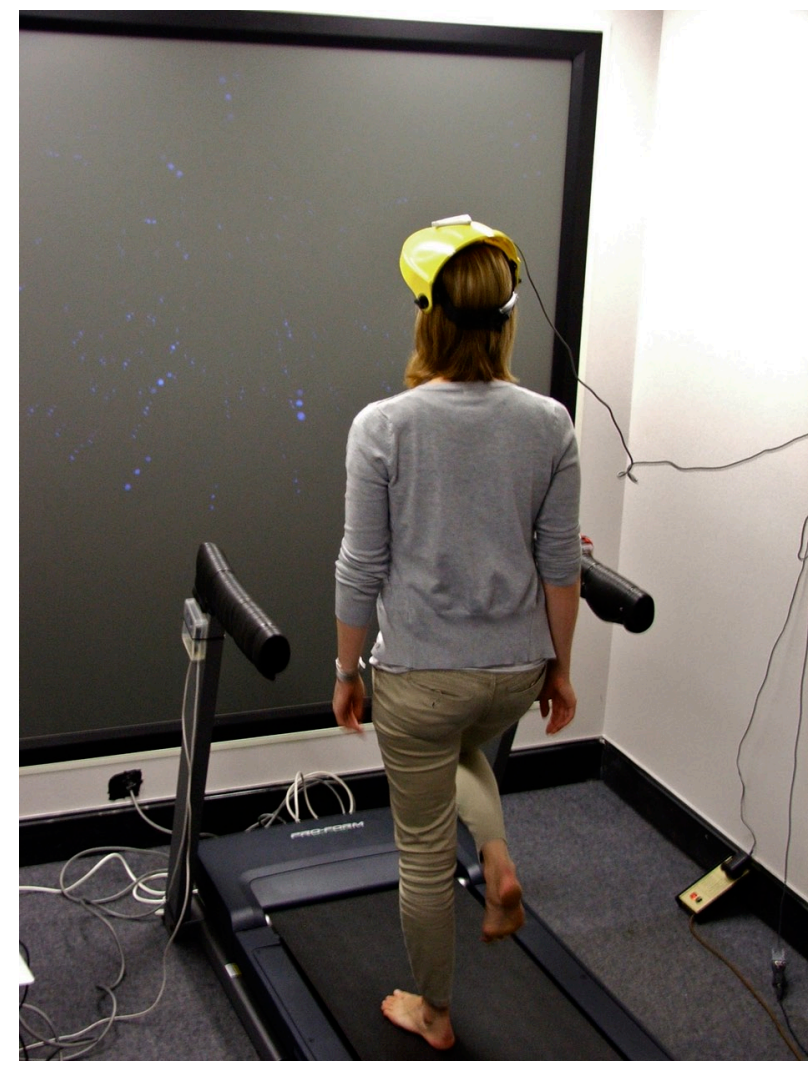

Figure 1. [In colour online, see http://dx.doi.org/10.1068/p7449] The set-up for experiments 1 and 2. 
For safety reasons subjects also wore a ceiling-mounted safety harness (B-Safe) throughout the experiment for both active and stationary viewing conditions. At the beginning of each active self-motion trial subjects were asked to use the treadmill's handrails until they felt comfortable with the simulated treadmill speed. Once subjects were comfortable with the speed of the treadmill, they let go of the handrails and used them only if they felt uncomfortable or disoriented. During stationary viewing conditions subjects stood on a stationary treadmill and viewed a playback of their previous active block of trials (jittering displays representing self-motion at either $4 \mathrm{~km} \mathrm{~h}^{-1}$ or $5 \mathrm{~km} \mathrm{~h}^{-1}$ ), or a smooth radially expanding optic flow display.

Subjects were asked to give a verbal rating of their perceived strength of vection in depth at the end of each self-motion trial on a graphical rating scale that ranged from 0 to 100 . This rating was made relative to a standard reference stimulus that subjects were told represented a rating of 50.(1) The reference stimulus was a constant velocity expanding optic flow stimulus simulating self-motion at $4 \mathrm{~km} \mathrm{~h}^{-1}$ and was viewed on a motionless treadmill while subjects were stationary. A rating of 0 indicated no experience of vection (the visual display motion was attributed solely to object motion - ie stationary observer), and a rating of 100 indicated complete or saturated vection (visual display motion was attributed solely to self-motion-ie stationary surround).

2.1.3 Procedure and design. The experiment was a $2 \times 2 \times 2$ within-subjects fully factorial design, and a repeated-measures ANOVA was used to analyse the data. We examined the following three independent variables: (i) treadmill and/or display speed $\left(4 \mathrm{~km} \mathrm{~h}^{-1}\right.$ or $\left.5 \mathrm{~km} \mathrm{~h}^{-1}\right)$; (ii) display type (jittering or nonjittering displays); and (iii) subject activity (active treadmill walking or passive viewing while stationary). Both treadmill and/or display speed and display type were within-block variables, and subject activity was a between-blocks variable. The dependent variable was the perceived strength of vection in depth. In the practice phase subjects were given 1 or 2 training trials at both treadmill and/or display speeds (ie at $4 \mathrm{~km} \mathrm{~h}^{-1}$ and $5 \mathrm{~km} \mathrm{~h}^{-1}$ ) until they were comfortable with walking at each simulated speed. During the testing phase the subjects were run through 3 experimental blocks of trials (these varied in terms of display speed and display type) consisting of 2 active blocks with a passive block run in between. Each experimental block consisted of 4 trials (ie purely radial optic flow at $4 \mathrm{~km} \mathrm{~h}^{-1}$, purely radial optic flow at $5 \mathrm{~km} \mathrm{~h}^{-1}$, jittering optic flow at $4 \mathrm{~km} \mathrm{~h}^{-1}$, and jittering optic flow at $5 \mathrm{~km} \mathrm{~h}^{-1}$ ) that were randomly presented and lasted approximately $30 \mathrm{~s}$. Subjects viewed displays in a darkened room; as subjects were positioned $0.9 \mathrm{~m}$ away from the display screen (ie the screen subtended an area approximately 79 deg wide by $67 \mathrm{deg}$ high), the edges of the display would have been visible only within the subject's periphery, almost 40 deg from the centre of their visual field.

\subsection{Results}

Vection was induced by all of the experimental conditions tested, with only the rated strength of this experience found to vary.

2.2.1 Main effects. We found a significant main effect of subject activity $\left[F_{1,19}=18.66, p<0.001\right.$, $\eta_{\mathrm{p}}^{2}=0.50$ (see figure 2)], where passive playback conditions were shown to result in significantly stronger vection in depth ratings than active treadmill walking conditions.

(1) This is the standard method for measuring vection, which is based on Stevens's (1957) method of magnitude estimation. In his original method Stevens used a standard modulus stimulus (what we call the standard reference stimulus) on which subjective estimates were based. In the case of the current study the modulus was set at 50 . No vection would, of course, be represented by a rating of " 0 ". Although defining end points is not ideal, and could compress estimates, this method is commonly used for measuring vection (see Kim and Palmisano 2008, 2010; Palmisano et al 2000, 2003, 2008). 


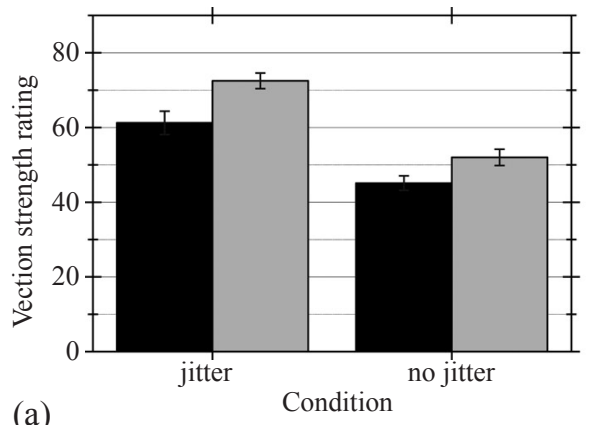

(a)

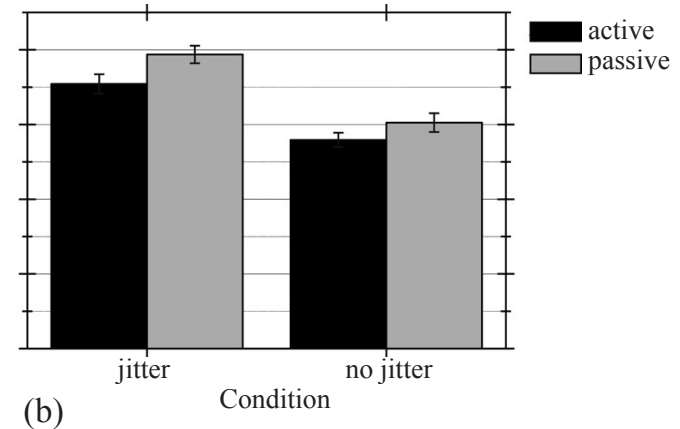

(b)

Figure 2. Effect of jittering and nonjittering displays during active walking and passive viewing conditions for treadmill and/or display specified forward speeds of (a) $4 \mathrm{~km} \mathrm{~h}^{-1}$ and (b) $5 \mathrm{~km} \mathrm{~h}^{-1}$ on vection in depth strength ratings (0-100). Error bars depict \pm 1 standard error of the mean.

We also found a significant main effect of display type $\left(F_{1,19}=46.03, p<0.001, \eta_{\mathrm{p}}^{2}=0.71\right)$ and of treadmill and/or display speed $\left(F_{1,19}=67.16, p<0.001, \eta_{\mathrm{p}}^{2}=0.78\right)$. Specifically, jittering displays and faster treadmill and/or display speeds $\left(5 \mathrm{~km} \mathrm{~h}^{-1}\right)$ were shown to produce significantly stronger vection in depth ratings than nonjittering displays and slower treadmill and/or display forward speeds $\left(4 \mathrm{~km} \mathrm{~h}^{-1}\right)$, respectively.

2.2.2 Interactions. There were no significant two-way or three-way interactions; however, we did find a trend toward an interaction between subject activity and display type [averaging across speed $\left(F_{1,19}=3.27, p=0.09, \eta_{\mathrm{p}}^{2}=0.15\right.$ (see figure 2$)$ ]. Specifically, as seen in figure 2 , there was a trend toward there being a greater difference in vection strength ratings between active and stationary viewing conditions for jittering self-motion displays than nonjittering self-motion displays.

\subsection{Discussion}

Contrary to Seno et al's (2011a) findings (but consistent with Onimaru et al 2010), we found that forward treadmill walking while viewing an expanding optic flow display reduced (rather than increased) vection in depth compared with viewing the same display while stationary. This reduction in vection in depth for active treadmill walking (compared with stationary viewing) was found irrespective of display type (jittering versus nonjittering displays) or treadmill and/or display simulated forward speed $\left(4 \mathrm{~km} \mathrm{~h}^{-1}\right.$ versus $\left.5 \mathrm{~km} \mathrm{~h}^{-1}\right)$. Overall, subjects' physical whole-body motion — which would provide vestibular, somatosensory, and proprioceptive information about self-motion - appeared to reduce vection compared with vision-only conditions.

Consistent with previous vection studies (see Palmisano et al 2011 for a review), we found a robust advantage for jittering self-motion displays compared with nonjittering constant velocity self-motion displays - this was true irrespective of whether the subject was walking on the treadmill or was physically stationary when viewing these displays. However, vection in depth was generally reduced - for both jittering and nonjittering self-motion displays alike - during treadmill walking compared with viewing these same displays while stationary.

We also found an advantage for faster treadmill and/or display-based simulated forward speeds compared with slower treadmill and/or display-based forward speeds. Consistent with our predictions and the findings of previous vection studies (Apthorp and Palmisano 2012; Brandt et al 1973; Palmisano 2002), faster forward display speeds increased vection in depth compared with slower forward display speeds. Our vection findings, however, showed no difference in reductions for vection during treadmill walking (compared with stationary 
viewing) between our two treadmill belt speeds. When matched to their respective display speeds, these two treadmill belt speeds resulted in comparable reductions in vection in depth strength ratings during treadmill walking (compared with stationary viewing).

Our findings could support a modified version of Barlow's (1990) inhibition theory (where the inhibition is assumed to apply not only to visually perceived object/scene motion but also to visually perceived self-motion). Consistent with Barlow, visual information about self-motion might have been inhibited/suppressed during forward treadmill walking conditions because subjects were viewing an expanding optic flow display (simulating forward self-motion), which would have provided redundant multisensory information about forward self-motion. To further test this possibility, in experiment 2 we compared this specific redundant self-motion situation with the following nonredundant self-motion situation: viewing a contracting optic flow display during forward treadmill walking.

\section{Experiment 2: the effect of simulated display direction on vection in depth during forward treadmill walking}

This experiment tested whether the observed vection reductions in experiment 1 for active treadmill walking (relative to stationary viewing conditions) were due to these displays specifying redundant multisensory information about self-motion. The conditions were identical to those in experiment 1 , with one exception: we also examined contracting optic flow (simulating backward self-motion) while subjects walked forward on a treadmill (ie a situation that provides nonredundant multisensory self-motion information). If reductions in vection in depth during treadmill walking are due to displays specifying redundant multisensory information about self-motion, then viewing an expanding optic flow display while walking forward on the treadmill might result in greater reductions in vection in depth (compared with vection in depth while stationary) than viewing a contracting optic flow display under the same conditions.

\subsection{Method}

3.1.1 Subjects. Fourteen psychology students (nine females and five males; mean age $=23.17$ years, $\mathrm{SD}=3.42$ years) at the University of Wollongong received course credit for their participation in this experiment. These were different subjects who met the same selection criteria as experiment 1 .

3.1.2 Design. The experiment was a $2 \times 2 \times 2 \times 2$ fully factorial design, and a repeatedmeasures ANOVA was used to analyse our data. There were four independent variables tested, including: (i) display type (jittering or nonjittering displays); (ii) subject activity (treadmill walking or passive viewing); (iii) treadmill and/or display speed $\left(4 \mathrm{~km} \mathrm{~h}^{-1}\right.$ or $5 \mathrm{~km} \mathrm{~h}^{-1}$ ); and (iv) display direction (contracting optic flow or expanding optic flow). Display type and 'treadmill and/or display' speed both varied within blocks, and subject activity and display direction both varied between blocks. As in our earlier experiments, the dependent variable was the perceived strength of vection in depth (rated on a scale of 0 to 100 compared with a reference stimulus - a constant velocity expanding optic flow display viewed while stationary — which was rated 50). It should be noted that during active conditions the simulated viewpoint jitter was always consistent with the subjects' actual head motion (irrespective of whether the display was also simulating forwards or backwards constant velocity self-motion).

\subsection{Results}

Similar to experiment 1 , vection was induced by all of the experimental conditions testedwith only the rated strength of this experience being found to vary. 
3.2.1 Main effects. Similar to experiment 1, we found a significant main effect of subject activity on vection $\left[F_{1,13}=33.13, p<0.001, \eta_{\mathrm{p}}^{2}=0.72\right.$ (see figure 3 )], where passively viewed self-motion displays resulted in significantly stronger vection in depth ratings than viewing displays during forward treadmill walking. Also consistent with experiment 1 , we found a significant main effect of display type $\left(F_{1,13}=100.58, p<0.001, \eta_{\mathrm{p}}^{2}=0.89\right)$ and treadmill and/or display speed $\left(F_{1,13}=49.52, p<0.001, \eta_{\mathrm{p}}^{2}=0.79\right)$. Specifically, jittering self-motion displays and faster treadmill/display-based simulated forward speeds $\left(5 \mathrm{~km} \mathrm{~h}^{-1}\right)$ resulted in significantly stronger vection in depth ratings than nonjittering self-motion displays and slower treadmill/display-based simulated speeds $\left(4 \mathrm{~km} \mathrm{~h}^{-1}\right)$, respectively. We did not find a significant main effect for display direction $\left(F_{1,13}=2.50, p=0.14\right)$.

3.2.2 Interactions. We found a significant three-way interaction between subject activity, display direction, and display type $\left[F_{1,13}=4.76, p=0.05, \eta_{\mathrm{p}}^{2}=0.27\right.$ (see figure 3 )]. To further examine this three-way interaction, we performed simple interaction effects (ie $2 \times 2$ repeated-measures ANOVAs) for each level of display type (jittering self-motion displays versus constant velocity self-motion displays).

When we performed a two-way ANOVA for nonjittering displays, we found a significant main effect of subject activity $\left(F_{1,13}=11.19, p=0.01, \eta_{\mathrm{p}}^{2}=0.46\right)$, but no main effect of display direction $\left(F_{1,13}=0.15, p=0.71\right)$, and no interaction between the two $\left(F_{1,13}=0.64\right.$, $p=0.44)$. These findings suggest that, irrespective of the simulated display direction, viewing nonjittering self-motion displays during treadmill walking resulted in reduced vection in depth compared with viewing these displays while stationary.

A two-way ANOVA for jittering displays showed a significant main effect of subject activity $\left(F_{1,13}=35.87, p<0.001, \eta_{\mathrm{p}}^{2}=0.73\right)$ and a significant main effect of display direction $\left(F_{1,13}=5.13, p=0.04, \eta_{\mathrm{p}}^{2}=0.28\right)$ as well as a significant interaction between the two $\left(F_{1,13}=9.57, p=0.01, \eta_{\mathrm{p}}^{2}=0.42\right)$. Simple effect contrasts for the interaction showed that there was: (i) no significant difference between expanding and contracting optic flow

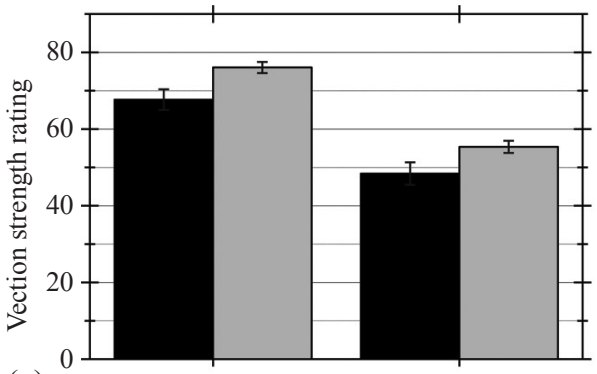

(a)

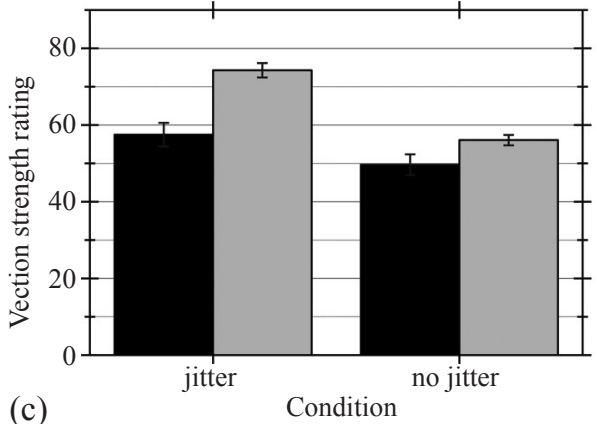

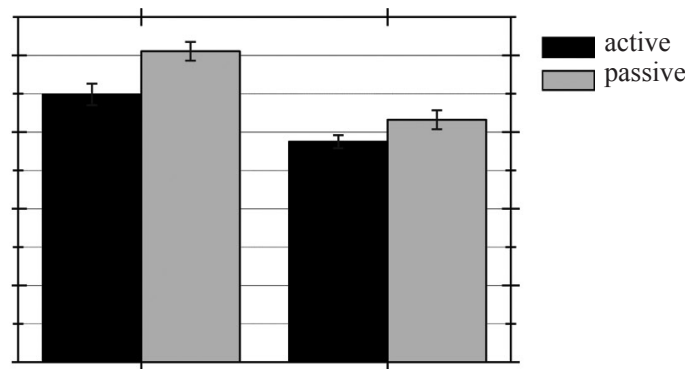

(b)

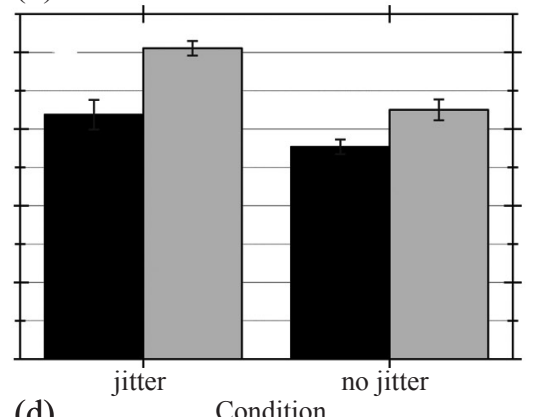

(d)

Figure 3. The effect of display type (jittering or nonjittering) and subject activity (active treadmill walking or passive viewing) for expanding optic flow displays simulated at (a) $4 \mathrm{~km} \mathrm{~h}^{-1}$ or (b) $5 \mathrm{~km} \mathrm{~h}^{-1}$ or contracting optic flow displays simulated at (c) $4 \mathrm{~km} \mathrm{~h}^{-1}$ or (d) $5 \mathrm{~km} \mathrm{~h}^{-1}$ on vection in depth strength ratings (0-100). Error bars depict \pm 1 standard error of the mean. 
for stationary viewing conditions $(p>0.05)$; and (ii) a significant difference between expanding and contracting optic flow for active treadmill walking conditions $(p<0.05)$. Specifically, the examination of means showed that jittering expanding optic flow produced stronger vection in depth ratings than jittering contracting optic flow displays. Therefore, viewing a jittering expanding optic flow display produced stronger vection in depth than viewing a jittering contracting optic flow display during forward treadmill walking (but not when viewing these same displays while stationary).

In addition to this three-way interaction, we found significant two-way interactions for: (i) display direction and display type $\left(F_{1,13}=7.10, p=0.02\right)$; (ii) subject activity and display type $\left(F_{1,13}=6.04, p=0.03\right)$; and (iii) subject activity and display direction $\left(F_{1,13}=5.93\right.$, $p=0.03)$. Consistent with the results of the three-way interaction, Bonferroni-corrected a posteriori contrasts (controlling for the family-wise error rate at 0.05 ) showed: (i) contracting and expanding optic flow resulted in significantly stronger vection than expanding optic flow displays in jittering conditions $\left(F_{1,13}=5.13, p=0.04\right)$, but no significant difference between contracting and expanding optic flow for nonjittering optic flow displays conditions; (ii) passive viewing conditions resulted in significantly stronger vection than active viewing conditions for jittering self-motion displays $\left(F_{1,13}=35.87, p<0.001\right)$, and passive nonjittering displays also resulted in significantly stronger vection than active conditions for nonjittering displays $\left(F_{1,13}=11.19, p=0.01\right)$; and, lastly, (iii) contracting optic flow displays resulted in significantly stronger vection than expanding optic flow displays for active conditions $\left(F_{1,13}=4.76, p=0.05\right)$, but no difference was found between contracting and expanding optic flow displays for passive conditions $\left(F_{1,13}=0.03, p=0.87\right)$.

\subsection{Discussion}

As in experiment 1 vection was reduced when subjects viewed expanding optic flow displays while walking forward on the treadmill (compared with when they viewed the same displays when stationary). However, vection was also reduced in a very similar fashion when subjects viewed contracting optic flow displays while walking forward on the treadmill. Thus, vection reductions during treadmill walking were not restricted to conditions which provided redundant information about self-motion. Treadmill walking reduced vection irrespective of whether the biomechanical self-motion stimulation was consistent or not with the visual self-motion stimulation.

As noted in the methods, simulated viewpoint jitter was always consistent with the subject's physical head movements in the active treadmill walking conditions, irrespective of the direction of self-motion simulated by the main (constant) velocity component of the optic flow. Adding this ecological simulated viewpoint jitter to inducing displays was found to enhance the vection induced in all of the different situations tested here (ie redundant treadmill walking, nonredundant treadmill walking, and viewing optic flow displays while stationary). Interestingly, this simulated viewpoint jitter was found to improve vection more when the biomechanical stimulation signaled the same direction of self-motion as the visual display.

\section{General discussion}

In our experiments forward treadmill walking always reduced the vection in depth induced by both jittering and nonjittering self-motion displays (compared with viewing these displays while stationary). These vection reductions during forward treadmill walking were very similar irrespective of the visually simulated direction of self-motion (ie forward or backward). Despite global reductions in vection for treadmill walking: (i) faster treadmill belt speeds and/or simulated forward self-motions $\left(5 \mathrm{~km} \mathrm{~h}^{-1}\right)$ were always found to produce stronger vection in depth ratings than slower treadmill belt speeds and simulated forward self-motions 
$\left(4 \mathrm{~km} \mathrm{~h}^{-1}\right)$; and (ii) jittering displays were always shown to produce stronger vection in depth ratings compared with constant velocity displays - irrespective of whether the subject viewed the display while walking or standing still. Importantly, these findings appear to show that reductions in vection in depth during forward treadmill walking (compared with stationary viewing) resulted from the act of, or the stimulation produced by, treadmill walking itself, rather than from the visual consequences of treadmill walking (ie as a result of the simulated head movements generated during treadmill walking).

Our findings do not appear to support a modified version of Barlow's (1990) inhibition theory for redundant or positively correlated sensory events during active self-motion. Here, we showed that forward treadmill walking reduced vection induced by both expanding (ie redundant information) and contracting (nonredundant or inconsistent information) optic flow displays (compared with viewing these same displays while stationary). Therefore, it appears that reductions in vection during forward treadmill walking (compared with stationary viewing conditions) are not exclusive to positively correlated and/or redundant multisensory situations. These vection reductions can also occur during negatively correlated and nonredundant multisensory situations.

The current findings of vection reductions during treadmill walking appear inconsistent with neurophysiological studies (Brandt et al 1998; Kleinschmidt et al 2002) and behavioural studies (Berthoz et al 1975; Wong and Frost 1981) reporting a reciprocal inhibitory interaction between the visual and nonvisual senses for self-motion. The reciprocal inhibition hypothesis proposes that, depending on the nature and type of stimulation, the most appropriate sense (typically vision) for a given situation may dominate the perception of self-motion and/or vection and downplay or attenuate information provided by the other subordinate sense(s). If vision was dominating this experience, then according to this hypothesis, conflicting nonvisual information should have had little to no effect on vection; but instead we found that conflicting biomechanical information about the direction of self-motion reduced vection during treadmill walking (compared with consistent biomechanical information about this experience). On the other hand, if nonvisual information was dominant, then we might have expected this situation to have inhibited (at least occasionally) the induction of vection during treadmill walking (as this experience relies on the domination of vision over the other senses). Contrary to this proposal, subjects experienced vection in all conditions, and it was only the strength of this experience that varied between conditions.

One reviewer suggested that our vection strength measure might have actually been indexing either perceived display speed (as in Durgin and Gigone 2007; Durgin et al 2005 studies) or vection speed. While we are confident that subjects were rating vection (we carefully instructed them how to rate the strength of their vection in both experiments and thoroughly checked this understanding again on debriefing), vection speed and vection strength have been shown to be positively related (Apthorp and Palmisano 2012; Palmisano 2002). Thus, we cannot rule out the possibility that vection speed (as opposed to strength) was a factor. It is even possible that simultaneous perceptions of object or scene motion and self-motion during treadmill walking (eg due to display lag) might have reduced the overall vection speed (eg assuming that the optic flow display was being perceptually parsed into components arising from separate self and object motions during these walking conditions). ${ }^{(2)}$ While it might be possible to explain the current vection reductions during treadmill walking by invoking notions of display or vection speed subtraction (eg Durgin 2009), these current findings may reflect a completely different phenomenon to visually perceived reductions in display speed also observed during treadmill walking.

(2) Note that we have shown that it is still possible to induce very compelling vection in physically stationary observers, even when they are simultaneously perceiving dramatic illusory scene shearingsee Palmisano et al (2006). 
Global reductions in vection during treadmill walking could be explained by attention. A study by Seno et al (2011b) showed that increases in attentional load reduced vection, suggesting that the induction of vection might require attentional resources. It is possible that, during treadmill walking, subjects were devoting attentional resources to staying upright on the moving treadmill, which could have detracted from the visually induced illusion of selfmotion. Studies have also suggested the possible influence of top-down, cognitive effects on multisensory integration where selective attention to an individual sense might attenuate the integration (and, thus, possible benefit) of consistent multisensory information (Mozolic et al 2008; Welch and Warren 1980). Therefore, it is possible that not only were subjects devoting attentional resources to treadmill walking but also that this attention generated a bias toward biomechanical information about self-motion and attenuated the integration of visual information about this self-motion experience. However, in contrast, studies by Kitazati and Sato (2003) and Trutoiu et al (2008) have shown the opposite effect, such that vection is enhanced when subjects were not paying attention to the vection-inducing stimulus, and inhibited when they were. On the basis of the above findings, we might have expected treadmill walking to have improved (rather than reduced) vection compared with stationary viewing self-motion situations. Future research needs to reconcile these contradictory findings to determine whether attention might improve or reduce vection during treadmill walking.

Finally, it is important to contemplate reasons for the apparent discrepancies between our current findings and those of Onimaru et al (2010) and Seno et al (2011a). It is possible that the discrepancy between our findings and Onimaru et al's (2010) findings were due to differences in the display or treadmill forward speeds between these two studies. Onimaru et al found a reduction in vection only when subjects were walking in the same direction as the simulated display motion (but not when subjects walked in the opposite direction to the simulated display motion). However, they used only a very slow treadmill belt speed $\left(2 \mathrm{~km} \mathrm{~h}^{-1}\right)$ that was below the range of normal human walking speeds. On the basis of the current findings, it is possible that global reductions for vection during forward treadmill walking occur only for common/typical human walking speeds. Furthermore, vection reductions during forward treadmill walking might be restricted to display speeds that are matched to the treadmill belt speed. Therefore, it is also possible that Seno et al (2011a) found an enhancement for expanding optic flow during forward treadmill walking (compared with contracting optic flow and stationary viewing) because the simulated display speed $\left(57.6 \mathrm{~km} \mathrm{~h}^{-1}\right)$ was much faster than the physical treadmill belt speed $\left(2 \mathrm{~km} \mathrm{~h}^{-1}\right)$. Examination of the simulated/physical speed of the display and the treadmill belt and how well they are 'matched' are both potentially promising avenues for reconciling previous findings for vection during treadmill walking.

In conclusion, our findings show that forward treadmill walking results in a global reduction in vection in depth compared with stationary viewing conditions. Unlike previous findings for perceived visual object speed, these vection reductions during forward treadmill walking were not exclusive to positively correlated multisensory self-motion situations; negatively correlated self-motion situations (ie viewing contracting optic flow) also generated an overall reduction in vection. Interestingly, however, contracting optic flow further reduced vection in depth compared with expanding optic flow for jittering (but not nonjittering constant velocity) displays - suggesting that the inconsistent biomechanical information was more disruptive during jittering constant velocity conditions. Consistent with previous seated vection studies, despite overall vection reductions for forward treadmill walking, jittering self-motion displays and faster display/treadmill forward speeds always facilitated vection compared with nonjittering self-motion displays and slower display/treadmill forward speeds, respectively. Overall, our findings suggest that: (i) biomechanical information downplays 
the overall visual experience of self-motion (and, thus, vection), particularly if it conflicts with visual information about the direction of self-motion during jittering displays; and (ii) consistent visual-vestibular information (provided by head-and-display motion) about self-motion always increases vection irrespective of the simulated direction of self-motion.

\section{References}

Apthorp D, Palmisano S, 2012 "Perceived display speed helps account for the 'jitter advantage' for vection" Perception 41 ECVP Supplement, 95 [abstract]

Ash A, Palmisano S, 2012 "Vection during conflicting multisensory information about the axis, magnitude, and direction of self-motion" Perception 41 253-267

Ash A, Palmisano S, Govan D, Kim J, 2011a "Display lag and gain effects on vection experienced by active observers" Aviation, Space and Environmental Medicine 82 763-769

Ash A, Palmisano S, Kim J, 2011b "Vection in depth during consistent and inconsistent multisensory stimulation" Perception 40 155-174

Barlow H B, 1990 "A theory about the functional role of synaptic mechanism of visual after-effects", in Vision: Coding and Efficiency Ed. C Blakemore (Cambridge: Cambridge University Press) pp 363-375

Benson A J, 1990 "Sensory functions and limitations of the vestibular system", in The Perception and Control of Self-motion Eds R Warren, A H Wertheim (Hillsdale, NJ: Lawrence Erlbaum Associates) pp 154-157

Berthoz A, Lacour M, Soechting J F, Vidal P P, 1979 "The role of vision in the control of posture during linear motion" Progress in Brain Research 50 197-209

Berthoz A, Pavard B, Young L R, 1975 "Perception of linear horizontal self-motion induced by peripheral vision (linear vection)" Experimental Brain Research 23 471-489

Brandt T, Bartenstein P, Janek A, Dieterich M, 1998 "Reciprocal inhibitory visual-vestibular interaction: Visual motion stimulation deactivates the parieto-insular vestibular cortex" Brain 121 1749-1758

Brandt T, Dichgans J, Koenig C, 1973 "Differential effects of central versus peripheral vision on egocentric and exocentric motion perception" Experimental Brain Research 16 476-491

Dichgans J, Brandt T, 1978 "Visual-vestibular interaction: Effects on self-motion perception and control", in Handbook of Sensory Physiology volume 8 Perception Eds R Held, H Leibowitz, H L Teuber (New York: Springer) pp 755-804

Diener H C, Dichgans J, Bruzek W, Selinka H, 1982 "Stabilization of human posture during induced oscillation of the body" Experimental Brain Research 45 126-132

Durgin F H, 2009 "When walking makes perception better" Current Directions in Psychological Science $1843-47$

Durgin F H, Gigone K, 2007 "Enhanced optic flow speed discrimination while walking: Contextual tuning in visual coding" Perception 36 1465-1475

Durgin F H, Gigone K, Scott R, 2005 "Perception of visual speed while moving" Journal of Experimental Psychology: Human Perception and Performance 31 339-353

Howard I P, 1982 Human Visual Orientation (Chichester, Sussex: John Wiley)

Howard I P, 1986 "The perception of posture, self-motion and visual vertical", in Handbook of Perception and Human Performance Volume 1, Eds K R Boff, L Kaufman, J P Thomas (New York: John Wiley) pp 18.1-18.62

Johansson G, 1977 "Studies on visual perception of locomotion" Perception 6 365-376

Kim J, Palmisano S, 2008 "Effects of active and passive viewpoint jitter on vection in depth" Brain Research Bulletin 77 335-342

Kim J, Palmisano S, 2010 "Visually mediated eye movements regulate the capture of optic flow in self-motion perception" Experimental Brain Research 202 355-361

Kitazati M, Sato T, 2003 "Attentional modulation of self-motion perception" Perception 32 475-484

Kleinschmidt A, Thilo K V, Büchel C, Gresty M A, Bronstein A M, Frackowiak R S J, 2002 "Neural correlates of visual motion perception as object- or self-motion" NeuroImage 16 873-882

Lee D N, Lishman J R, 1975 "Visual proprioceptive control of stance" Journal of Human Movement Studies $187-95$ 
Li L, Adelstein B D, Ellis S R, 2009 "Perception of image motion during head movement" $A C M$ Transactions of Applied Perception 6(1) article 5

Lishman J R, Lee D N, 1973 “The autonomy of visual kinaesthesis” Perception 2 287-294

Melvill-Jones G, Young L R, 1978 "Subjective detection of vertical acceleration: A velocity dependent response" Acta Oto-laryngologica 85 45-53

Mergner T, Rosemeier T, 1998 "Interaction of vestibular, somatosensory and visual signals for postural control and motion perception under terrestrial and microgravity conditions - a conceptual model" Brain Research Reviews 28 118-135

Mozolic J L, Hugenschmidt C E, Peiffer A M, Laurienti P J, 2008 “Modality-specific selective attention attenuates multisensory integration" Experimental Brain Research 184 39-52

Onimaru S, Sato T, Kitazaki M, 2010 "Veridical walking inhibits vection perception" Journal of Vision 10(7):860 [abstract]

Palmisano S, 2002 "Consistent stereoscopic information increases the perceived speed of vection in depth" Perception 31 463-480

Palmisano S, Allison R S, Howard I P, 2006 "Illusory scene distortion occurs during perceived selfrotation in roll" Vision Research $\mathbf{4 6} 4048-4058$

Palmisano S, Allison R S, Kim J, Bonato F, 2011 "Simulated viewpoint jitter shakes sensory conflict accounts of vection" Seeing and Perceiving 24 173-200

Palmisano S, Allison R S, Pekin F, 2008 “Accelerating self-motion displays produce more compelling vection in depth" Perception $3722-33$

Palmisano S, Burke D, Allison R S, 2003 "Coherent perspective jitter induces visual illusions of selfmotion" Perception 32 97-110

Palmisano S, Gillam B J, Blackburn S G, 2000 "Global-perspective jitter improves vection in central vision" Perception 29 57-67

Seno T, Ito H, Sunaga S, 2011a "Inconsistent locomotion inhibits vection" Perception 40 747-750

Seno T, Ito H, Sunaga S, 2011b "Attentional load inhibits vection” Attention, Perception, \& Psychophysics 73 1467-1476

Siegler I, Viaud-Delmon I, Israël I, Berthoz A, 2000 "Self-motion perception during a sequence of whole-body rotations in darkness" Experimental Brain Research 134 66-73

Souman J L, Freeman T C, Eikmeier V, Ernst M O, 2010 "Humans do not have direct access to retinal flow during walking" Journal of Vision 10(11):14, 1-12

Stevens S S, 1957 “On the psychophysical law” Psychological Review 64 153-181

Trutoiu L C, Streuber S, Mohler B J, Schulte-Pelkum J, Bülthoff H H, 2008 "Tricking people into feeling like they are moving when they are not paying attention", in Proceedings of the 5th Symposium on Applied Perception in Graphics and Visualization page 190

Walsh E G, 1961 "Role of the vestibular apparatus in the perception of motion on a parallel swing" Journal of Physiology 155 506-513

Welch R B, Warren D H, 1980 “Immediate perceptual response to intersensory discrepancy" Psychological Bulletin 88 638-667

Wong S C P, Frost B J, 1981 "The effect of visual-vestibular conflict on the latency of steady-state visually induced subjective rotation" Perception \& Psychophysics $30228-236$

Yeung K L, Li L, 2013 "Effect of the field of view on perception world-referenced image motion during concurrent head movements" Displays 34 165-170

Zacharias G L, Young L R, 1981 "Influence of combined visual and vestibular cues on human perception and control of horizontal rotation" Experimental Brain Research 41 159-171 